\title{
Quantum Monte Carlo study of the energetics of the rutile, anatase, brookite, and columbite $\mathrm{TiO}_{2}$ polymorphs
}

\author{
John Trail,,${ }^{1, *}$ Bartomeu Monserrat,,${ }^{1,2}$ Pablo López Ríos, ${ }^{1}$ Ryo Maezono, ${ }^{3}$ and Richard J. Needs ${ }^{1}$ \\ ${ }^{1}$ Theory of Condensed Matter Group, Cavendish Laboratory, \\ J. J. Thomson Avenue, Cambridge CB3 OHE, UK \\ ${ }^{2}$ Department of Physics and Astronomy, Rutgers University, Piscataway, New Jersey 08854-8019, USA \\ ${ }^{3}$ School of Information Science, JAIST, Asahidai 1-1, Nomi, Ishikawa 923-1292, Japan
}

(Dated: September 17, 2018)

\begin{abstract}
The relative energies of the low-pressure rutile, anatase, and brookite polymorphs and the highpressure columbite polymorph of $\mathrm{TiO}_{2}$ have been calculated as a function of temperature using the diffusion quantum Monte Carlo (DMC) method and density functional theory (DFT). The vibrational energies are found to be important on the scale of interest and significant quartic anharmonicity is found in the rutile phase. Static-lattice DFT calculations predict that anatase is lower in energy than rutile, in disagreement with experiment. The accurate description of electronic correlations afforded by DMC calculations and the inclusion of anharmonic vibrational effects contribute to stabilizing rutile with respect to anatase. Our calculations predict a phase transition from anatase to rutile $\mathrm{TiO}_{2}$ at $630 \pm 210 \mathrm{~K}$.
\end{abstract}

PACS numbers: 02.70.Ss, 63.20.Ry, 91.60.Ed

Titanium dioxide $\left(\mathrm{TiO}_{2}\right)$ finds many applications in photocatalysis $[1,2]$ and as a catalyst support [3]. It exhibits high chemical and optical stability, long-term durability, corrosion resistance, low cost and non-toxicity. Two main polymorphs of $\mathrm{TiO}_{2}$ occur in nature, rutile and anatase [4], and a third polymorph, brookite, also exists at ambient conditions [5]. Several high-pressure forms of $\mathrm{TiO}_{2}$ have also been synthesized, the most stable of which is columbite $\left(\mathrm{TiO}_{2}-\mathrm{II}\right)[6]$.

Experiment and extrapolations from experimental data suggest that rutile is the most stable phase from 0 to above $1300 \mathrm{~K}$ [7]. Anatase and brookite are observed to undergo irreversible phase transitions to the rutile form at high temperatures $[7,8]$, indicating the presence of high energetic barriers between different polymorphs. Although this could suggest that anatase and brookite are metastable at all temperatures, the high energetic barriers make it difficult to determine phase transition temperatures between polymorphs. This suggests that accurate computations of the relative stabilities of the polymorphs could provide valuable insights into the phase stability of the $\mathrm{TiO}_{2}$ system.

Relative energies of $\mathrm{TiO}_{2}$ polymorphs have been calculated in numerous first-principles density-functionaltheory (DFT) electronic structure studies [9-17], which suggest that the three low-pressure polymorphs, rutile, anatase, and brookite, are close in energy under ambient conditions, although the results obtained depend significantly on the density functional used, and are therefore inconclusive.

The main goal of the present work is to estimate the relative energies of rutile, anatase, brookite and columbite $\mathrm{TiO}_{2}$ with as high an accuracy as possible. We calculate the relative electronic energies of $\mathrm{TiO}_{2}$ polymorphs using the diffusion quantum Monte Carlo (DMC) method [18, 19], which is the most accurate method known for calculating the energy of a large system of quantum particles. Furthermore, we find that the energetic contribution arising from thermal nuclear motion, which we have calculated using DFT, is crucial in determining the phase stability of $\mathrm{TiO}_{2}$ polymorphs, and that anharmonic terms are important in dynamically stabilizing the rutile polymorph. A very recent study has reported similar results, although anharmonic vibrations were not considered [20].

The quantum Monte Carlo calculations were performed using the CASINO code [21]. The cost of a DMC calculation scales approximately as the cube of the number of particles $N$ for fermionic systems, which allows applications to large systems. The central approximation in a DMC calculation is the "fixed-node constraint" [22]. The nodal surface is the $(3 N-1)$-dimensional surface on which the wave function is zero and across which it changes sign. A trial wave function is optimized using the variational quantum Monte Carlo (VMC) method, and the nodal surface of the DMC wave function is constrained to equal that of the trial wave function. An importance-sampling transformation is employed in DMC which ensures that the important parts of the wave function are sampled most often, and reduces the fluctuations in the energy. In DMC the imaginary-time Schrödinger equation is used to evolve an ensemble of electronic configurations towards the ground state distribution. VMC and DMC are variational techniques in the sense that they give an energy that is higher than or equal to the exact energy, subject to a small statistical error that can be systematically reduced by running the simulations for longer. The variational property of VMC and DMC aids cancellation of errors in energy differences. 
We used a trial wave function of Slater-Jastrow form

$$
\Psi_{\mathrm{SJ}}(\mathbf{R})=\exp [J(\mathbf{R})] \operatorname{det}\left[\psi_{n}\left(\mathbf{r}_{i}^{\uparrow}\right)\right] \operatorname{det}\left[\psi_{n}\left(\mathbf{r}_{j}^{\downarrow}\right)\right],
$$

where $\mathbf{R}$ denotes the positions of all of the electrons, $\mathbf{r}_{i}^{\uparrow}$ is the position of the $i$ th spin-up electron, $\mathbf{r}_{j}^{\downarrow}$ is the position of the $j$ th spin-down electron, $\exp [J(\mathbf{R})]$ is a Jastrow correlation factor $[23,24]$, and $\operatorname{det}\left[\psi_{n}\left(\mathbf{r}_{i}^{\uparrow}\right)\right]$ and $\operatorname{det}\left[\psi_{n}\left(\mathbf{r}_{i}^{\downarrow}\right)\right]$ are determinants of up- and down-spin single-particle orbitals. The DMC calculations were performed using the T-moves scheme which ensures that the energy remains greater than the ground-state energy when pseudopotentials are used [25-27].

Single-particle orbitals for the $\mathrm{TiO}_{2}$ structures were calculated using the CASTEP plane-wave DFT code [28], the PBEsol functional [29] and a large basis-set energy cutoff of 160 Ry $(\simeq 2177 \mathrm{eV})$, which provided DFT energy differences between structures converged to within $0.0036 \mathrm{eV} /\left[\mathrm{TiO}_{2}\right]$ of the large basis set limit. The orbitals were transformed into a "blip" polynomial basis for efficient evaluation in the calculations [30]. We used Jastrow factors consisting of an electron-nucleus term represented by a polynomial of order 8 , an electron-electron term represented by a polynomial of order 8 , and a cosine expansion with 4 inequivalent parameters per spin-pair type, giving a total of 43 optimizable parameters. The wavefunction parameters were optimized using VMC, with the variance of the energy minimized first [31], followed by minimization of the variational energy $[32,33]$.

We used correlated-electron pseudopotentials (CEPPs) for $\mathrm{O}$ [34] and $\mathrm{Ti}[35]$ generated from ab initio multiconfigurational Hartree-Fock (MCHF) atomic calculations $[36,37]$. The CEPPs give significantly more accurate results than Hartree-Fock pseudopotentials [38, 39]. CEPPs contain two-body operators that describe core polarization [40] and their high accuracy has been verified in tests using coupled cluster calculations including single, double, and perturbative triple excitations $[\operatorname{CCSD}(\mathrm{T})][41]$ with the Molpro code [42]. The O CEPP has a He core and the $d$ channel was chosen as local. A projector was generated from the ground state orbitals for both the $s$ and $p$ channels. The Ti pseudopotential has a Ne core and the $f$ channel was chosen as local. The semi-core nature of the Ti CEPP was captured using five projectors. We use the highest angular momentum channel available as the local potential, which helps to reduce the errors associated with using pseudopotentials in DMC [27].

The thermodynamic limit was approached in our calculations by using large simulation cells containing 768 electrons (32 formula units) subject to periodic boundary conditions. We used experimental cell parameters and atomic positions at $300 \mathrm{~K}$ from Refs. 43 (rutile), 44 (anatase), 5 (brookite), and 45 (columbite). Cell dimensions, and the number of formula units contained within each of them, are given in Table I. We chose simulation cells that maximize the distance between periodic images [46], which mitigates finite-size errors [47, 48].

DMC calculations were performed for each structure at a single wavevector which was chosen from the wavevectors that yield a real-valued wave function so as to minimize the difference between the DFT energy at the single wavevector and the converged DFT energy. We used the finite-size correction scheme of Kwee et al. [49] in which an alternative local density approximation (LDA) functional (the "KZK" functional, constructed from DMC data for homogeneous electron gases) provides reference DFT energies that include finite-size effects, which can then be used to construct a finite-size correction for the DMC energies.

Our main results were obtained using a DMC time step $\tau$ of 0.004 a.u. and a target population of 10,240 configurations. While explicit time-step extrapolation is common practice [50], test DMC calculations with time steps of $\tau=0.001,0.0025$ and 0.004 a.u. using simulation cells containing 192 electrons (8 formula units) showed that time-step errors in energy differences were negligible at $\tau=0.004$ a.u., validating the use of a single time step. More details of the finite-size corrections and extrapolations are provided in the Supplemental Material [51].

Figure 1 shows the energies of the four polymorphs obtained in DMC and for DFT calculations using the same set of functionals used to assess the accuracy of the DMC finite-size corrections [29, 52-56]. Energies are given relative to that of anatase for each method. Calculations with "on-the-fly" CASTEP DFT pseudopotentials [28] produced similar results to those shown in Fig. 1.

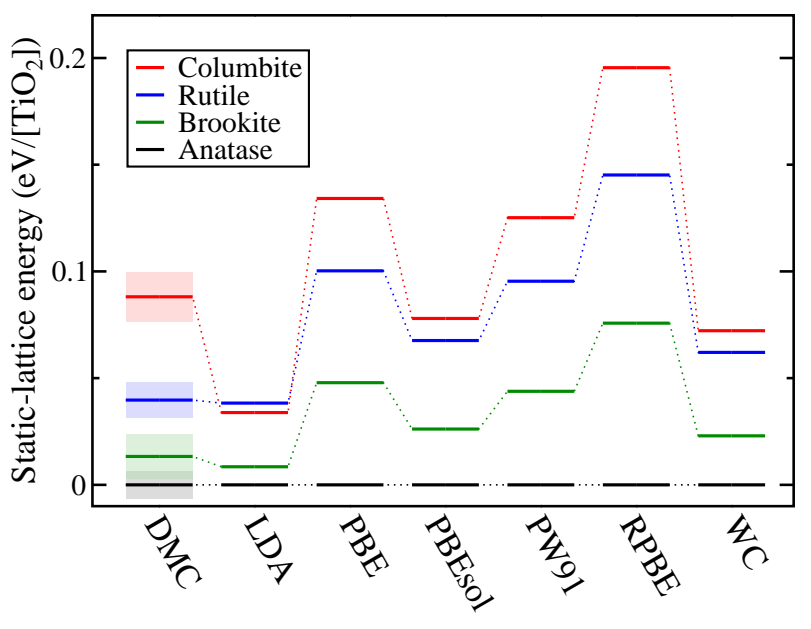

FIG. 1. Static-lattice energies relative to that of anatase from DMC and DFT calculations using the experimental lattice constants. The statistical errors in the DMC results are represented by translucent rectangles.

Given the relatively large variation of structural energies with the choice of functional and the estimated errors for DMC results, these data show that the DFT level of theory provides an inadequate description of the relative 


\begin{tabular}{llllcll}
\hline \hline & \multicolumn{2}{c}{ Unit-cell lattice constants $(\AA)$} & formula & \multicolumn{2}{c}{$F(300 \mathrm{~K})\left(\mathrm{eV} /\left[\mathrm{TiO}_{2}\right]\right)$} \\
Polymorph & $a$ & $b$ & $c$ & units & DMC & DFT \\
\hline Rutile [43] & 4.5922 & 4.5922 & 2.9574 & 2 & $-2476.228(8)$ & -2470.2631 \\
Anatase [44] & 3.7845 & 3.7845 & 9.5143 & 4 & $-2476.246(6)$ & -2470.3091 \\
Brookite [5] & 9.174 & 5.449 & 5.138 & 8 & $-2476.24(1)$ & -2470.2826 \\
Columbite [45] & 4.61 & 5.43 & 4.87 & 4 & $-2476.15(1)$ & -2470.2254 \\
\hline \hline
\end{tabular}

TABLE I. Experimental orthorhombic unit cells used for the four $\mathrm{TiO}_{2}$ polymorphs. The Helmholtz free energies at $300 \mathrm{~K}$, $F(300 \mathrm{~K})$, estimated as the sum of electronic energy and the anharmonic vibrational free energy at $300 \mathrm{~K}$ are also shown. The anharmonic vibrational free energy is calculated using the PBEsol functional and VSCF equations, whereas the electronic energy is evaluated with both DMC and DFT using the PBEsol functional.

energies of the four structures. The generalized gradient approximation (GGA) functionals were found to provide the same energetic ordering of the phases as DMC, but most, with the notable exception of the PBEsol and WC functionals, tend to overestimate the energy differences, while the LDA functional significantly underestimates the static-lattice energy of columbite. The relative DFT energies are expected to show significant self-interaction errors which give rise to unphysical delocalization of the $3 d$ electrons.

The inclusion of nuclear vibrational motion provides significant corrections to the static-lattice energies of $\mathrm{TiO}_{2}$ polymorphs. We have performed vibrational calculations for the four polymorphs at the anharmonic vibrational level using a vibrational self-consistent-field (VSCF) method [57] and the PBEsol density functional. The vibrational calculations were performed using the efficient "nondiagonal supercells" method [58] which allows us to fully converge the results with respect to system size.

The inclusion of anharmonic contributions in our lattice dynamics calculations is motivated by the presence of unstable vibrational modes in rutile $\mathrm{TiO}_{2}$ when described within the harmonic approximation [59-61]. Using the experimental volume, we find unstable modes within various regions of the vibrational Brillouin zone (BZ), mostly around the $\Gamma$ point and the $\left(\frac{1}{2}, \frac{1}{2}, \frac{1}{4}\right)$ point. The presence of unstable modes is independent of the density functional used to perform the calculations (see Supplemental Material [51]). Instead of directly including anharmonic effects, first-principles DFT calculations for $\mathrm{TiO}_{2}$ have mostly been performed using structures that are relaxed to volumes which are smaller than the experimental volume, an approach that tends to remove the dynamical instabilities but leads to a substantial bias in the energy differences between structures.

Figure 2 shows a slice through the Born-Oppenheimer (BO) energy surface of rutile $\mathrm{TiO}_{2}$. The atomic configurations at which we have evaluated the $\mathrm{BO}$ energies are along a line spanned by one of the two soft modes at the $\Gamma$ point. Our VSCF calculations show that the inclusion of anharmonicity leads to dynamical stability throughout the $\mathrm{BZ}$, arising primarily from quartic terms in the BO potential. Figure 2 illustrates the quartic anharmonicity of the BO energy surface and the resulting vibrational density. The explicit inclusion of anharmonicity plays a significant role in the energetics of rutile $\mathrm{TiO}_{2}$, with the anharmonic free energy differing from harmonic estimates of the free energy by up to $0.035 \mathrm{eV} /\left[\mathrm{TiO}_{2}\right]$ (see Supplemental Material [51]).

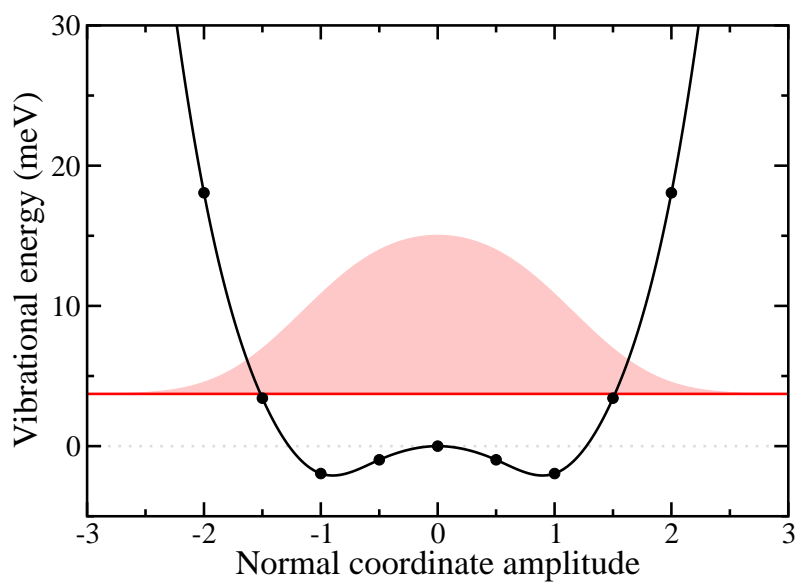

FIG. 2. A slice through the Born-Oppenheimer energy surface at the $\Gamma$ point of the Brillouin Zone. The red shaded area shows the vibrational density for a mode that is unstable at the harmonic level but is stabilized by anharmonicity. The horizontal red line shows the energy of the mode. The normal coordinate is measured in units of $1 / \sqrt{2|\omega|}$, where $\omega$ is the imaginary harmonic frequency of the mode.

We take the Helmholtz free energy to be the sum of the static-lattice electronic energy and the anharmonic vibrational free energy at $300 \mathrm{~K}$, which is a valid approximation due to the large band gap of $\mathrm{TiO}_{2}$ polymorphs relative to the thermal energy. Figure 3 shows the total static-lattice energies and the Helmholtz free energies at $300 \mathrm{~K}$ using electronic energies evaluated with DMC and DFT using the PBEsol functional. The DMC Helmholtz free energies of anatase, brookite, and rutile are indistinguishable within our target accuracy of 0.01 $\mathrm{eV} /\left[\mathrm{TiO}_{2}\right]$. Our final DFT and DMC Helmholtz free energies at $300 \mathrm{~K}$ including the effects of anharmonic vibrations are summarized in Table I.

We also evaluate the Helmholtz free energy at a range of temperatures, using the $300 \mathrm{~K}$ experimental unit cells. 


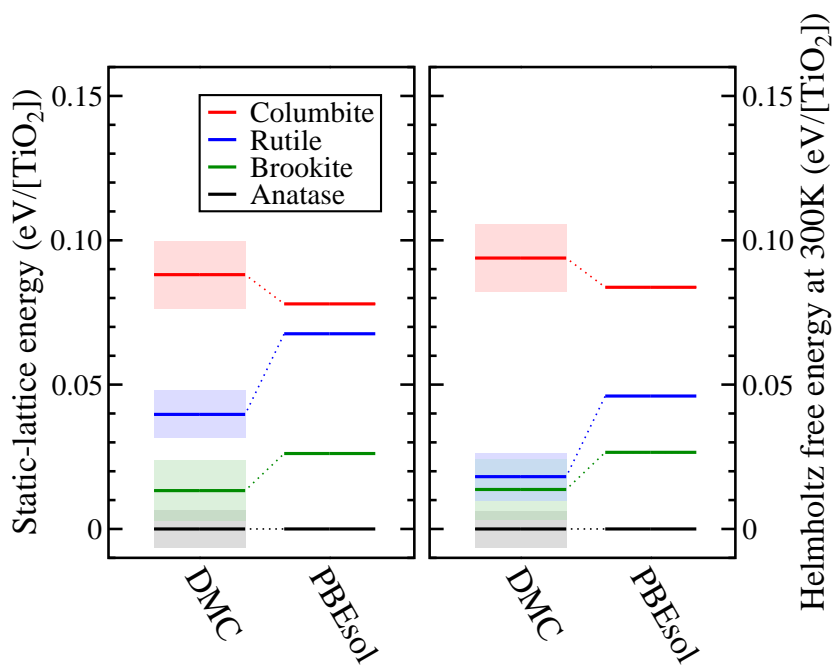

FIG. 3. Left: static-lattice energies relative to that of anatase from DMC and DFT-PBEsol calculations, and right: Helmholtz free energies at $300 \mathrm{~K}$ evaluated by adding DFTbased vibrational corrections to the DMC and DFT-PBEsol energies. The statistical error in the DMC results is represented by translucent rectangles. The DFT data were generated using a k-point grid spacing of $0.060 \AA^{-1}$ with an error estimated to be less than $0.004 \mathrm{eV} /\left[\mathrm{TiO}_{2}\right]$.

The resulting Helmholtz free energies, relative to anatase, are shown in Fig. 4, suggesting a phase transition from anatase to rutile at $630 \pm 210 \mathrm{~K}$. We estimate the error incurred by neglecting thermal expansion in our calculations to be less than $0.003 \mathrm{eV} /\left[\mathrm{TiO}_{2}\right]$ over the temperature range $300-575 \mathrm{~K}$ by comparing the $\mathrm{PBEsol}$ harmonic free energies of anatase and rutile $\mathrm{TiO}_{2}$ at the fixedvolume geometries with those at the variable-volume experimental unit cell geometries provided by Hummer et al. [62].

Our results using the experimental geometries at $300 \mathrm{~K}$ for all temperatures predict the stability of anatase at low temperatures. However, the experimentally observed rutile polymorph is energetically very close to being stable, which strongly suggests that reproducing the experimental results requires both an accurate description of electronic correlation and anharmonic vibrations.

There are several sources of error in our calculations. The DMC energies are affected by time-step and population-control bias, although we have verified that these effects are negligible compared with our target accuracy of $0.01 \mathrm{eV} /\left[\mathrm{TiO}_{2}\right]$. We have made every effort to minimize the fixed-node errors, which we expect to be small, although they are difficult to quantify. We have employed finite-size corrections using methods that have been demonstrated to be accurate and consistent with direct extrapolation [46]. Similarly, while pseudopotentials are inherently approximate, our tests have shown that CEPPs provide very accurate representations of the $\mathrm{Ti}$ and $\mathrm{O}$ atoms. The use of pseudopotentials in DMC in-

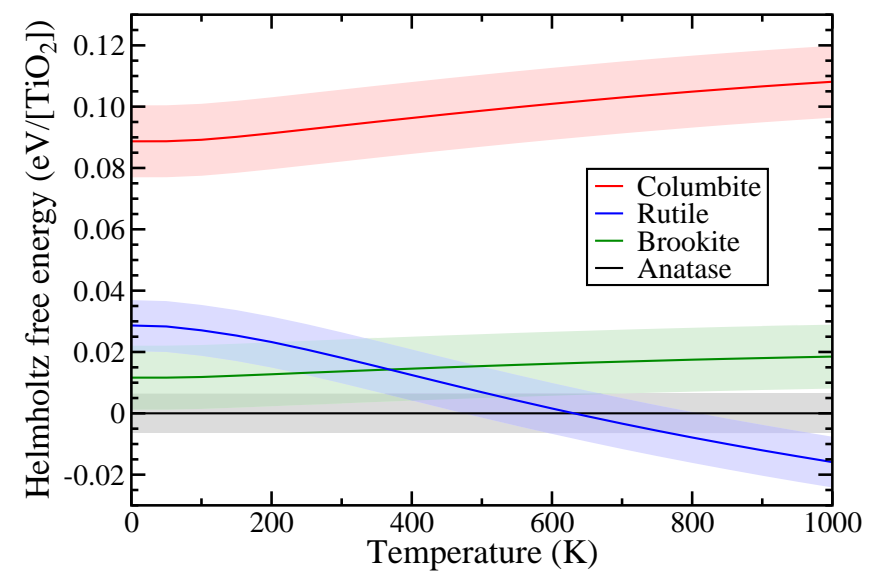

FIG. 4. Helmholtz free energies relative to that of anatase as a function of temperature. These are estimated as the sum of the DMC electronic energy and the DFT anharmonic vibrational free energy at each temperature. Both the electronic energies and the anharmonic vibrational free energies are evaluated for $300 \mathrm{~K}$ experimental unit cells at all temperatures. Statistical errors are represented by translucent regions. Details of the DFT anharmonic vibrational energy calculations are as in Fig. 3.

curs an additional bias, but the T-moves scheme ensures that this bias is positive, which promotes cancellation of errors in energy differences.

We suggest that the disagreement with experiment is likely to arise mainly from the DFT evaluation of vibrational properties. DFT calculations with both the LDA and PBE density functionals have been reported to underestimate harmonic frequencies in diamond [63], and the size of this underestimation is of the order of magnitude of the difference between the Helmholtz free energies of anatase and rutile $\mathrm{TiO}_{2}$ at low temperatures reported in our work.

In summary, we have obtained accurate estimates of the relative energies of rutile, anatase, brookite, and columbite $\mathrm{TiO}_{2}$ using DMC methods, and of the relative Helmholtz free energies by combining static-lattice DMC methods and anharmonic vibrational methods. The main calculations were performed using DMC static-lattice calculations, and finite-size corrections were obtained from the KZK scheme. Our results confirm that columbite $\mathrm{TiO}_{2}$ is significantly higher in energy than the three lowpressure phases. The lowest (anatase) and highest (rutile) Helmholtz free energies of the low-pressure phases at $300 \mathrm{~K}$ differ by only about $0.02 \mathrm{eV} /\left[\mathrm{TiO}_{2}\right]$, which is close to our target accuracy of $0.01 \mathrm{eV} /\left[\mathrm{TiO}_{2}\right]$. We have shown that an accurate description of both electronic correlation and vibrational energies are required to provide accurate energetics for the $\mathrm{TiO}_{2}$ polymorphs considered. Our VSCF calculations show that the rutile structure of $\mathrm{TiO}_{2}$ is stabilized by quartic anharmonic vibrational motion. Our results are consistent with the experimen- 
tally observed irreversible phase transitions of anatase and brookite to the rutile form at high temperatures, but do not explain the stability of rutile at low temperatures $[7,8]$.

J.R.T., P.L.R., and R.J.N. acknowledge financial support from the Engineering and Physical Sciences Research Council (EPSRC) of the U.K. under grant number EP/J017639/1. B.M. acknowledges Robinson College, Cambridge, and the Cambridge Philosophical Society for a Henslow Research Fellowship. R.M. is grateful for financial support from MEXT-KAKENHI grants 26287063, 25600156, 22104011, and a grant from the Asahi Glass Foundation. Computational resources were provided by the Archer facility of the U.K.'s national high-performance computing service (for which access was obtained via the UKCP consortium, grant number $\mathrm{EP} / \mathrm{K} 014560 / 1)$, by the Center for Information Science of the JAIST, and by the K-computer (supported by the Computational Materials Science Initiative, CMSI/Japan, under project numbers hp120086, hp140150, and hp150014). Preliminary calculations were performed using the Darwin Supercomputer of the University of Cambridge High Performance Computing Service (http://www.hpc.cam.ac.uk). Supporting research data may be freely accessed at [URL], in compliance with the applicable Open Data policies.

* jrt32@cam.ac.uk

[1] Y. Bai, I. Mora-Sero, F. De Angelis, J. Bisquert, and P. Wang, Chem. Rev. 114, 10095 (2014).

[2] K. Hashimoto, H. Irie and A. Fujishima, Jpn. J. Appl. Phys. 44, 8269 (2005).

[3] K. Bourikas, C. Kordulis, and A. Lycourghiotis, Chem. Rev. 114, 9754 (2014).

[4] D. A. H. Hanaor and C. C. Sorrel, J. Mater. Sci. 46, 855 (2011).

[5] E. P. Meagher and G. A. Lager, Can. Mineral. 17, 77 (1979).

[6] J. C. Jamieson, and B. Olinger, Am. Mineral. 54, 1477 (1969).

[7] S. J. Smith, R. Stevens, S. Liu, G. Li, A. Navrotsky, J. Boerio-Goates, and B. F. Woodfield, Am. Mineral. 94, 236 (2009).

[8] Y. Aoki and S. Saito, JPS Conf. Proc. 4, 012005 (2015).

[9] M. Lazzeri, A. Vittadini, and A. Selloni, $\quad$ Phys. Rev. B 63, 155409 (2001); Erratum Phys. Rev. B 65, 119901(E) (2002).

[10] M. E. Arroyo-de Dompablo, A. Morales-Garcia, and M. Taravillo, J. Chem. Phys. 135, 054503 (2011).

[11] N. H. Vu, H. V. Le, T. M. Cao, V. V. Pham, H. M. Le, and D. Nguyen-Manh, J. Phys.: Condens. Matter 24, 405501 (2012).

[12] F. De Angelis, C. Di Valentin, S. Fantacci, A. Vittadini, and A. Selloni, Chem. Rev. 114, 9708 (2014).

[13] J. Muscat, V. Swamy, and N. M. Harrison, Phys. Rev. B 65, 224112 (2002).

[14] L. Beltran, L. Gracia, and J. Andres,
J. Phys. Chem. B 110, 23417 (2006).

[15] M. Landmann, E. Rauls, and W. G. Schmidt, J. Phys.: Condens. Matter 24, 195503 (2012).

[16] A. C. Withers, E. J. Essene, Y. Zhang, Contrib. Mineral. Petrol. 145, 199 (2003).

[17] T. Zhu and S.-P. Gao, J. Phys. Chem. C 118, 11385 (2014).

[18] D. M. Ceperley and B. J. Alder, Phys. Rev. Lett. 45, 566 (1980).

[19] W. M. C. Foulkes, L. Mitas, R. J. Needs, and G. Rajagopal, Rev. Mod. Phys. 73, 33 (2001).

[20] Y. Luo, A. Benali, L. Shulenburger, J. T. Krogel, O. Heinonen, and P. R. C. Kent, arXiv:1607.07361.

[21] R. J. Needs, M. D. Towler, N. D. Drummond, and P. López Ríos, J. Phys.: Condens. Matter 22, 023201 (2010).

[22] J. B. Anderson, J. Chem. Phys. 65, 4121 (1976).

[23] N. D. Drummond, M. D. Towler, and R. J. Needs, Phys. Rev. B 70, 235119 (2004).

[24] P. López Ríos, P. Seth, N. D. Drummond, and R. J. Needs, Phys. Rev. E 86, 036703 (2012).

[25] M. Casula, Phys. Rev. B 74, 161102 (2006).

[26] M. Casula, S. Moroni, S. Sorella, and C. Filippi, J. Chem. Phys. 132, 154113 (2010).

[27] N. D. Drummond, J. R. Trail, and R. J. Needs, arXiv:1607.01544 (to be published).

[28] S. J. Clark, M. D. Segall, C. J. Pickard, P. J. Hasnip, M. I. J. Probert, K. Refson, and M. C. Payne, Z. Kristallogr. 220, 567 (2005).

[29] J. P. Perdew, A. Ruzsinszky, G. I. Csonka, O. A. Vydrov, G. E. Scuseria, L. A. Constantin, X. Zhou, and K. Burke, Phys. Rev. Lett. 100, 136406 (2008); Erratum Phys. Rev. Lett. 102, 039902(E) (2009).

[30] D. Alfe and M. J. Gillan, Phys. Rev. B 70, 161101 (2004).

[31] N. D. Drummond and R. J. Needs, Phys. Rev. B 72, 085124 (2005).

[32] C. J. Umrigar, J. Toulouse, C. Filippi, S. Sorella, and R. G. Hennig, Phys. Rev. Lett. 98, 110201 (2007).

[33] J. Toulouse and C. J. Umrigar, J. Chem. Phys. 126, 084102 (2007).

[34] J. R. Trail and R. J. Needs, J. Chem. Phys. 139, 014101 (2013).

[35] J. R. Trail and R. J. Needs, J. Chem. Phys. 142, 064110 (2015).

[36] C. F. Fischer, G. Tachiev, G. Gaigalas, and M. Godefroid, Comput. Phys. Commun. 176, 559 (2007).

[37] A. Borgoo, O. Scharf, G. Gaigalas, and M. Godefroid, Comput. Phys. Commun. 181, 426 (2010).

[38] J. R. Trail and R. J. Needs, J. Chem. Phys. 122, 174109 (2005).

[39] J. R. Trail and R. J. Needs, J. Chem. Theory Comput. 10, 2049 (2014).

[40] Y. Lee and R. J. Needs, Phys. Rev. B 67, 035121 (2003).

[41] K. Raghavachari, G. W. Trucks, J. A. Pople, and M. Head-Gordon, Chem. Phys. Lett. 157, 479 (1989).

[42] H.-J. Werner et al., Computational Chemistry and Molecular Modelli

[43] R. J. Swope, J. R. Smyth, and A. C. Larson, Am. Mineral. 80, 448 (1995).

[44] C. J. Howard, T. M. Sabine and F. Dickson, Acta Crystallogr. B 47, 462 (1991).

[45] S. Y. Chen and P. Shen, Phys. Rev. Lett. 89, 096106 (2002).

[46] N. D. Drummond, B. Monserrat, J. H. Lloyd-Williams, 
P. López Ríos, C. J. Pickard, and R. J. Needs, Nat. Commun. 6, 7794 (2015)

[47] N. D. Drummond, R. J. Needs, A. Sorouri, and W. M. C. Foulkes, Phys. Rev. B 78, 125106 (2008); Erratum Phys. Rev. B 90, 159901(E) (2014).

[48] P. R. C. Kent, R. Q. Hood, A. J. Williamson, R. J. Needs, W. M. C. Foulkes, and G. Rajagopal, Phys. Rev. B 59, 1917 (1999).

[49] H. Kwee, S. Zhang, and H. Krakauer, Phys. Rev. Lett. 100, 126404 (2008).

[50] R. M. Lee, G. J. Conduit, N. Nemec, P. López Ríos, and N. D. Drummond, Phys. Rev. E 83, 066706 (2011); Erratum Phys. Rev. E 85, 029908(E) (2012).

[51] See the Supplemental Material at [URL] for details of the DFT and DMC calculations, including discussion of pseudopotentials, finite-size effects, time step errors, and the harmonic and anharmonic vibrational calculations.

[52] J. P. Perdew and A. Zunger, Phys. Rev. B 235048 (1981).

[53] J. P. Perdew, K. Burke and M. Ernzerhof, Phys. Rev. Lett. 77, 3865 (1996).

[54] J. P. Perdew, J. A. Chevary, S. H. Vosko, K. A. Jack- son, M. R. Pederson, D. J. Singh and C. Fiolhais, Phys. Rev. B 46, 6671 (1992).

[55] B. Hammer, L. B. Hansen and J. K. Norskov, Phys. Rev. B 59, 7413 (1999).

[56] Z. Wu and R. E. Cohen, Phys. Rev. B 73, 235116 (2006).

[57] B. Monserrat, N. D. Drummond, and R. J. Needs, Phys. Rev. B 87, 144302 (2013).

[58] J. H. Lloyd-Williams and B. Monserrat, Phys. Rev. B 92, 184301 (2015).

[59] P. D. Mitev, K. Hermansson, B. Montanari, and K. Refson, Phys. Rev. B 81, 134303 (2010).

[60] T. Lan, C. W. Li, O. Hellman, D. S. Kim, J. A. Munoz, H. Smith, D. L. Abernathy, and B. Fultz, Phys. Rev. B 92, 054304 (2015).

[61] B. Wehinger, A. Bosak, and P. T. Jochym, Phys. Rev. B 93, 014303 (2016).

[62] D. R. Hummer, P. J. Heaney, and J. E. Post, Powder Diffr. 22, 10095 (2007).

[63] R. Maezono, A. Ma, M. D. Towler, and R. J. Needs, Phys. Rev. Lett. 98, 025701 (2007). 